\title{
Nodes selection strategy in cooperative tracking problem ${ }^{1}$
}

\author{
Wen Yang ${ }^{\mathrm{a}}$, Zidong Wang ${ }^{\mathrm{b}}$, Zongyu Zuo ${ }^{\mathrm{c}}$, Chao Yang ${ }^{\mathrm{a}}$, Hongbo Shi ${ }^{\mathrm{a}}$ \\ ${ }^{a}$ Key Laboratory of Advanced Control and Optimization for Chemical Processes (East China University of Science and Technology), \\ Ministry of Education, Shanghai, China \\ ${ }^{\mathrm{b}}$ Department of Computer Science, Brunel University London, Uxbridge, Middlesex, UB8 3PH, U.K.
}

${ }^{\mathrm{c}}$ The Seventh Research Division, Science and Technology on Aircraft Control Laboratory, Beihang University, Beijing, China

\begin{abstract}
In this paper, a new optimization problem is addressed for node selection that has application potentials in input/output switches for sensors in control system design and leader determination in social networks. The purpose of the addressed problem is to develop a strategy for selecting a subset of nodes as controlled nodes in order to minimize certain objective function consisting of the convergence speed and the energy of control action, over a finite time-horizon. For networks with fixed controlled nodes, an upper bound of the objective function is obtained which is shown to be convex and independent of the time-horizon. For networks with switched controlled nodes, a greedy algorithm is proposed to reduce the computation complexity resulting from the length of the time-horizon, where the nodes selection is carried out over divided small time-intervals. The cost gap is also analyzed between the strategy of optimizing over the whole time-horizon and the strategy of optimizing over the small intervals. Finally, the proposed nodes selection strategy is validated through simulations and two regions are found in which the number of optimal controlled nodes is determined.
\end{abstract}

Key words: Cooperative control; Controlled consensus; Node selection; Convex optimization.

\section{Introduction}

Consensus-based dynamical networks have proven to be an effective yet flexible framework for modeling the multiagent information sharing problems involving cooperative tasks with examples including wireless sensor navigation, spacecraft formation control, mobile robot rendezvous and unmanned aerial vehicle flocking. The last decade has witnessed surged research interest on the consensus problem and a rich body of results has been reported in the literature, see e.g. [1]- [9]. In recent years, particular research efforts have been devoted to the controllability properties of the underlying interaction network in order to provide a systematic way for characterizing/designing useful interaction models, see [10]- [20] for some representative results. For example, Rahmani et al. [18] have shown how the symmetry structure of the network, characterized in terms of its automorphism group, is directly related to the controllability of the corresponding multi-agent system. In [19], Egerstedt et al. have discussed the relationship between the network structure and the controllability properties in single-leader consensus networks, and have also summarized some recent problems/results appearing in the past five years.

\footnotetext{
* This paper was not presented at any IFAC meeting. Corresponding author: Wen Yang.

Email addresses: weny@ecust. edu. cn (Wen Yang), Zidong.Wang@brunel.ac.uk (Zidong Wang),

zzybobby@buaa.edu. cn (Zongyu Zuo),

yangchaopku@gmail.com (Chao Yang),

hbshi@ecust. edu. cn (Hongbo Shi).
}

In parallel with the controllability problem of multi-agent systems, the nodes selection problem has been a research focus over the past few years due to the fact that a suitable selection of the nodes would have a major impact on the effectiveness of adopting control technologies in real-world applications. For example, in the area of control system design, it is vitally important to select appropriate input/output sensors so as to maintain the desired control performances. In social networks, needless to say, selecting the right leaders is the key to ensure efficient spreading of the information to the whole network. The nodes selection problem has been recently studied for leader-follower systems and many results have appeared. In [21], Porfiri et al. have proposed a node-to-node pinning control strategy to optimize the control performance, where the control input is added on a switched node each time. In [22], by using an $\mathrm{H}_{2}$ norm of the system, Patterson et al. have examined the effect of the leader selection on the coherence of the network which characterizes the level of agreement of the nodes if the external disturbances exist. In [23], Kawashima et al. have investigated the leader selection problem by using the manipulability index to measure the influence of leaders' inputs on the network centroid. Specifically, Andrew et al. [26] have studied leader selection in order to minimize convergence errors experienced by the follower agents, and Lin et al. [27] have looked into the leaders selection problem by minimizing the mean-square deviation from consensus in stochastically forced networks.

In this paper, we consider a class of cooperative tracking problem [28-31] with aim to select a subset of nodes (referred to as the controlled nodes) to be injected into the con- 
trol inputs so as to drive the remaining nodes to reach the desired consensus. Our attention is focused on how to select the controlled nodes in order to optimize some network performances. It is worth mentioning that the controlled nodes in the proposed system are affected by their adjacent nodes while the states of the leader nodes in the leader-follower system are fixed at the beginning. In most of the existing literature, the network performance index has been either the convergence speed or the network robustness against noises. Different from the existing literature, we propose a new network performance index described by a quadratic function that takes both the convergence speed and the energy of the control actions. Such a new index is motivated by the classical $L Q R$ problem that minimizes a cost function accounting for the trade-off between the undesired deviation and the energy incurred by the control action. In addition, we set a constraint on the number of the controlled nodes in order to take the resource limitation into consideration. Accordingly, we formulate the nodes selection problem as an optimization one that seeks a set of binary values indicating whether a node is selected to be the controlled node. In the case of selecting fixed nodes, we aim to select a set of nodes by minimizing the network performance index with an inequality constraint of the number of selected nodes. In the case of selecting switched nodes, we aim to select a set of nodes at each time step by minimizing the network performance index with the nodes number constraint at each time step and the constraint of using frequency of each node.

It is widely recognized that the main challenge in selecting nodes stems from the Boolean constraints which give rise to a combinatorial optimization problem. In this paper, we introduce an iterative method to relax the Boolean constraints to a convex hull by replacing the $l_{0}$ norm with the $l_{1}$ norm. The main contributions of this paper are highlighted as follows. 1) In the case when the controlled nodes are fixed, an upper bound of the objective function is derived which is shown to be independent of the time-horizon, and the addressed nodes selection problem is converted into a convex optimization one that can be readily solved by some standard methods such as the interior point algorithm. 2) In the case when the controlled nodes switch, a greedy algorithm is exploited to solve the nodes selection problem over many small time-intervals, thereby significantly reducing the computation complexity for large-scale networks over lengthy time-horizon. The relationships among the network parameters are discussed and the cost gap of the objective function is also examined between the original optimization strategy and the transformed optimization strategy with the greedy algorithm. 3) By simulations, a tradeoff is found to exist between the convergence speed and the energy of control actions, where the number of optimally controlled nodes is determined under certain conditions.

The remainder of this paper is organized as follows. In Section 2, we introduce the cooperative tracking problem and analyze the stability of the networked system with extra control inputs. In Section 3, we formulate the nodes selection as an optimization problem and propose some iterative selec- tion algorithms in the cases when the controlled nodes are fixed and switched. Finally, simulation examples are given in Section 4 and some concluding remarks are given in the end.

Notations: $\mathbb{R}^{n}$ is the $n$-dimensional Euclidean space. $\mathbb{S}^{n}$ represents the set of symmetric $n \times n$ matrices. $\mathbb{S}_{+}^{n}$ and $\mathbb{S}_{++}^{n}$ are the sets of symmetric positive semidefinite and positive definite matrices, respectively. When the matrix $X$ is positive semi-definite (positive definite), it is denoted as $X \succeq 0$ ( $X \succ$ $0)$. Moreover, $X \succeq Y$ if $X-Y$ is a positive semi-definite matrix. $\operatorname{tr}(\cdot)$ is the trace of a matrix. $I_{n}$ is the $n$-dimensional identity matrix. $\mathbf{1}_{n}$ is the vector with all components being 1. $\rho(\cdot)$ is the spectral radius of a matrix. $\operatorname{diag}(X)$ denotes the diagonal matrix with it diagonal blocks being $X$.

\section{Problem Formulation}

Consider a network described as an undirected graph $G=$ $(V, E)$ with $V=(1,2, \ldots, n)$ being the set of $n$ nodes and the edges $E \subset V \times V$ representing the communication links. Denote the set of neighbors of node $i$ by $N_{i}=\{j:(i, j) \in E\}$. Each node can exchange information with its neighbors. The interconnection topology of the network is described by a weighted matrix $W=\left[w_{i j}\right]$, where $w_{i i}=1-\sum_{j \in N_{i}} w_{i j}$ and $0<w_{i j}<1$ if $(i, j) \in E$; otherwise, $w_{i j}=0$. Here, we assume that the network $G$ is connected, and $W$ is thus an irreducible non-negative matrix.

In the network, each node updates its state as

$$
x_{i}(k+1)=\sum_{j=1}^{n} w_{i j} \cdot x_{j}(k)+l \cdot \gamma_{i}(k) \cdot u_{i}(k)
$$

where

$$
u_{i}(k)=c-x_{i}(k) .
$$

Here, $x_{i}(k) \in \mathbb{R}^{m}$ is the state of the $i$ th node at the time step $k, c \in \mathbb{R}^{m}$ is the desired state. Note that

$$
0<l<\min _{i}\left\{w_{i i}\right\}, 1 \leq i \leq n
$$

is a constant gain. If the $i$ th node is injected into the control input at time step $k$, then $\gamma_{i}(k)=1$, which is referred to as the controlled node; otherwise, $\gamma_{i}(k)=0$. In the case of selecting fixed nodes, the controlled nodes are fixed, i.e., $\gamma_{i}(k)$ is constant for all $k=1,2, \ldots$ In the case of selecting switched nodes, the controlled node switches at each time step $k$. To simplify the addressed problem, we set $m=1$. However, all the results can be extended to the case $m>1$ by using the Kronecker product.

By collecting all the states of the nodes, we define

$$
\begin{gathered}
x(k) \triangleq\left[x_{1}(k), \ldots, x_{n}(k)\right]^{\prime}, u(k) \triangleq\left[u_{1}(k), \ldots, u_{n}(k)\right]^{\prime}, \\
\Gamma(k) \triangleq \operatorname{diag}\left(\gamma_{1}(k), \ldots, \gamma_{n}(k)\right) .
\end{gathered}
$$


The single node dynamics in conjunction with the control inputs (2) can be represented as the following vector form

$$
x(k+1)=\hat{W}(k) x(k)+c l \cdot \Gamma(k) \mathbf{1}_{n},
$$

where $\hat{W}(k)=W-l \Gamma(k)$.

In this paper, we are interested in designing an algorithm to determine $\gamma_{i}(k)$ so that the networked system (3) achieves the desired agreement with satisfactory performance. First, we examine whether all the nodes converge to the desired state $c$ by adding the control inputs on a part of nodes.

It is worth mentioning that the reachability problem of the cooperative tracking protocol have been studied extensively. The following result follows from the existing results in $[18,26,27]$.

Proposition 1 Consider an undirected network consisting of $n$ nodes with dynamics (1). In the case of selecting fixed nodes, the states of all the nodes converge to the desired state if an arbitrary node is selected as the controlled node. In the case of selecting switched nodes, the states of all the nodes converge to the desired state if an arbitrary node is selected as the controlled node at each time step.

Proof: Under the assumption that $G$ is connected, $W$ is nonnegative and irreducible. By Proposition 4.1 in [18], it is easy to show that the dynamic system (1) achieves agreement asymptotically in both cases of fixed and switched nodes.

Remark 1 Note that the reachability of the cooperative tracking protocol (3) is independent of the number of the controlled nodes. Some existing works have shown that the number of the controlled nodes influences the convergence speed.

In the following, we endeavor to study the nodes selection problem by answering the following fundamental question: if the number of controlled nodes is less than a prescribed value, how to select a subset of nodes as the controlled nodes so as to minimize a desired objective function? We will approach the addressed problem in the following two cases.

Case 1. For the case of selecting fixed nodes, we formulate the problem as follows:

$$
\begin{aligned}
\left(P_{0}\right): \quad \min _{\Gamma} & J(\Gamma)=\sum_{k=0}^{T}\left(p\|x(k)\|_{2}^{2}+(1-p)\|u(k)\|_{2}^{2}\right) \\
\text { s.t. } \quad \gamma_{i} \in\{0,1\}, i=1, \ldots, n, & t r(\Gamma) \leq q, \\
& 0 \leq p \leq 1,
\end{aligned}
$$

where the objective function is composed by two terms, the first quadratic term $x(k)^{\prime} x(k)$ quantifies the energy consumed by the control action, and the second term quantifies the convergence speed. The reason why we choose this objective function is simply to consider the tradeoff between the convergence speed and the energy of the control actions in practice. Here, $p$ is the weighting factor and the number of controlled nodes is limited to be less than $q>0$. The diagonal matrix $\Gamma$ is the optimization variable whose diagonal elements are binary $\gamma_{i}$ 's, $i=1,2, \ldots, n$.

Case 2. In real-time applications, a node might be used for a finite number of times only because of the limited energy or the communication fault. Therefore, it is necessary to discuss the problem of selecting switched controlled nodes, which can be formulated as follows:

$$
\begin{aligned}
\left(P_{1}\right): \quad \min _{\Gamma(k)} & J(\Gamma(k))=\sum_{k=0}^{N T}\left(p\|x(k)\|_{2}^{2}+(1-p)\|u(k)\|_{2}^{2}\right) \\
\text { s.t. } \quad \gamma_{i}(k) \in\{0,1\}, i=1, \ldots, n, & \\
& \sum_{k=0}^{N T} \gamma_{i}(k) \leq r_{i}, \\
& \operatorname{tr}(\Gamma(k)) \leq q, \\
& 0 \leq p \leq 1,
\end{aligned}
$$

where each node can be used for less than $r_{i}$ times, see the second constraint above. Throughout the remainder of this paper, we denote the minimum value of $J$ by $J^{\star}$ when $\Gamma(k)$ is chosen (among all stabilizing controlled nodes) to yield the best achievable performance.

Notice that, due to the Boolean constraints of $\gamma_{i}(k)$ 's, $P_{0}$ and $P_{1}$ are actually combinatorial optimization problems which would require intractable search to obtain the solutions. Over the last decades, a variety of methods have been developed to solve the related combinatorial optimization problem. In this paper, we will introduce an iterative method to relax the Boolean constraints by using the reweighted $l_{1}$ norm.

\section{Main results}

As can be seen from Proposition 1, to achieve desired agreement, the selection of the controlled nodes is not unique. Choosing an optimal node set with minimum number of nodes among all the possibilities is crucial to the minimization of the objective function. In the following, we will show that the problem $P_{0}$ and $P_{1}$ can be relaxed to a convex optimization problem. In the case of selecting fixed nodes, we will derive an upper bound for the objective function which is independent of the time-horizon $T$. In the case of selecting switched nodes, we will use a method similar to the greedy algorithm to reduce the computation complexity, which solves the optimization problem over each of certain small time-intervals.

\subsection{Fixed nodes selection problem}

In this case, the controlled nodes are fixed during the whole time horizon and the selection matrix $\Gamma$ is time-invariant.

Letting $u(k)=c \mathbf{1}_{n}-x(k)$, we obtain

$$
u(k+1)=(W-l \Gamma) u(k)
$$


and, by iteration, we have

$$
u(k)=(W-l \Gamma)^{k} u(0)
$$

and then

$$
\begin{aligned}
\sum_{k=0}^{\infty} u(k)^{\prime} u(k) & =u(0)^{\prime} \sum_{k=0}^{\infty}\left(\hat{W}^{k}\right)^{\prime} \hat{W}^{k} u(0) \\
& =u(0)^{\prime}\left(I_{n}-\hat{W}^{2}\right)^{-1} u(0) .
\end{aligned}
$$

Since

$$
\left(I_{n}-\hat{W}^{2}\right)^{-1}=\frac{1}{2}\left(I_{n}+\hat{W}\right)^{-1}+\frac{1}{2}\left(I_{n}-\hat{W}\right)^{-1},
$$

then we have

$$
\sum_{k=0}^{\infty} u(k)^{\prime} u(k)=\frac{1}{2} u(0)^{\prime}\left[\left(I_{n}+\hat{W}\right)^{-1}+\left(I_{n}-\hat{W}\right)^{-1}\right] u(0)
$$

Here, the quadratic function $u(k)^{\prime} u(k)$ is convex because the inverse of a positive definite matrix is a convex function of the matrix (See Exercise 3.18, [34]). Now, we proceed to investigate the quadratic function $x(k)^{\prime} x(k)$. According to Proposition $4.1 \mathrm{in}$ [18], it is easy to show that $-I_{n} \prec \hat{W} \prec I_{n}$. By recursion, we have

$$
\begin{aligned}
x(k) & =\hat{W}^{k} x(0)+l \sum_{i=0}^{k-1} \hat{W}^{i} \Gamma c \mathbf{1}_{n}, \\
& =\hat{W}^{k} x(0)+l\left(I_{n}-\hat{W}^{k}\right)\left(I_{n}-\hat{W}\right)^{-1} \Gamma c \mathbf{1}_{n}, \\
& =\hat{W}^{k} x(0)+c\left(I_{n}-\hat{W}^{k}\right) \mathbf{1}_{n},
\end{aligned}
$$

where the last equality follows from the fact that

$$
l \Gamma \mathbf{1}_{n}=(W-\hat{W}) \mathbf{1}_{n}=\left(I_{n}-\hat{W}\right) \mathbf{1}_{n} .
$$

Then, we have

$$
\begin{aligned}
& \sum_{k=0}^{T} p\|x(k)\|_{2}^{2}+(1-p)\|u(k)\|_{2}^{2} \\
& =\sum_{k=0}^{T} p\left\|\hat{W}^{k} x(0)+c\left(I_{n}-\hat{W}^{k}\right) \mathbf{1}_{n}\right\|_{2}^{2} \\
& \quad+\frac{1}{2}(1-p) u(0)^{\prime}\left[\left(I_{n}+\hat{W}\right)^{-1}+\left(I_{n}-\hat{W}\right)^{-1}\right] u(0) .
\end{aligned}
$$

Next, we provide an upper bound for $\sum_{k=0}^{T}\|x(k)\|_{2}^{2}$, which is proved to be convex.

\section{Theorem 1 Consider the minimization problem}

$$
\begin{array}{ll}
\min _{\Gamma} & \sum_{k=0}^{T}\left\|\hat{W}^{k} x(0)+c\left(I_{n}-\hat{W}^{k}\right) \mathbf{1}_{n}\right\|_{2}^{2} \\
\text { s.t. } & \gamma_{i} \in\{0,1\}, i=1, \ldots, n .
\end{array}
$$

The suboptimal solution of (6) can be obtained by solving the following problem

$$
\begin{array}{ll}
\min _{\Gamma} & \frac{1}{2} x(0)^{\prime}\left[\left(I_{n}+\hat{W}\right)^{-1}+\left(I_{n}-\hat{W}\right)^{-1}\right] x(0) \\
\text { s.t. } & \gamma_{i} \in\{0,1\}, i=1, \ldots, n
\end{array}
$$

where the objective function in (7) is the upper bound of the objective function in (6).

Proof: Since $\rho(\hat{W})<1$, it is easy to obtain

$$
\begin{aligned}
\sum_{k=0}^{T} x(0)^{\prime}\left(\hat{W}^{k}\right)^{\prime} \hat{W}^{k} x(0) & <\sum_{k=0}^{\infty} x(0)^{\prime}\left(\hat{W}^{k}\right)^{\prime} \hat{W}^{k} x(0) \\
& =x(0)^{\prime}\left(I_{n}-\hat{W}^{2}\right)^{-1} x(0) .
\end{aligned}
$$

It follows from $\hat{W}^{2} \preceq \hat{W}$ that

$$
\begin{aligned}
\sum_{k=0}^{T}\left(I_{n}-\hat{W}^{k}\right)^{2} & =(1+T) I_{n}-2 \sum_{k=0}^{T} \hat{W}^{k}+\sum_{k=0}^{T} \hat{W}^{2 k} \\
& \preceq(1+T) I_{n}-\left(I_{n}+\hat{W}+\hat{W}^{2}+\ldots+\hat{W}^{T}\right) .
\end{aligned}
$$

Furthermore, it is easy to see

$$
\mathbf{1}_{n}^{\prime}(W-l \Gamma) \mathbf{1}_{n}=\mathbf{1}_{n}^{\prime} \mathbf{1}_{n}-\mathbf{1}_{n}^{\prime}(l \Gamma) \mathbf{1}_{n} .
$$

Noticing that $\Gamma$ is a nonnegative diagonal matrix, we have

$$
\begin{aligned}
\mathbf{1}_{n}^{\prime}(W-l \Gamma)^{2} \mathbf{1}_{n} & =\mathbf{1}_{n}^{\prime} \mathbf{1}_{n}+\mathbf{1}_{n}^{\prime}\left(l^{2} \Gamma^{2}-2 l \Gamma\right) \mathbf{1}_{n} \\
& >\mathbf{1}_{n}^{\prime} \mathbf{1}_{n}-\mathbf{1}_{n}^{\prime}(2 l \Gamma) \mathbf{1}_{n},
\end{aligned}
$$

and

$$
\begin{aligned}
\mathbf{1}_{n}^{\prime}(W-l \Gamma)^{3} \mathbf{1}_{n} & =\mathbf{1}_{n}^{\prime} \mathbf{1}_{n}-\mathbf{1}_{n}^{\prime}(3 l \Gamma) \mathbf{1}_{n}+\mathbf{1}_{n}^{\prime}\left(3 l^{2} \Gamma^{2}-l^{3} \Gamma^{3}\right) \mathbf{1}_{n} \\
& >\mathbf{1}_{n}^{\prime} \mathbf{1}_{n}-\mathbf{1}_{n}^{\prime}(3 l \Gamma) \mathbf{1}_{n}
\end{aligned}
$$

By induction method, we deduce that

$$
\mathbf{1}_{n}^{\prime}(W-l \Gamma)^{k} \mathbf{1}_{n}>\mathbf{1}_{n}^{\prime} \mathbf{1}_{n}-\mathbf{1}_{n}^{\prime}(k l \Gamma) \mathbf{1}_{n}
$$

Using all the previous arguments and $\mathbf{1}_{n}^{\prime} \Gamma \mathbf{1}_{n}=q$, we obtain

$$
\begin{aligned}
\mathbf{1}_{n}^{\prime} \sum_{k=0}^{T}\left(I_{n}-\hat{W}^{k}\right)^{2} \mathbf{1}_{n} & <\mathbf{1}_{n}^{\prime}(l \Gamma+2 l \Gamma+\ldots+l T \Gamma) \mathbf{1}_{n} \\
& =\frac{1}{2} l q T(T+1)
\end{aligned}
$$

Moreover, by using the elementary inequality $\left(\alpha^{\frac{1}{2}} x-\right.$ $\left.\alpha^{-\frac{1}{2}} y\right)\left(\alpha^{\frac{1}{2}} x-\alpha^{-\frac{1}{2}} y\right)^{\prime} \geq 0$ where $x$ and $y$ are row vectors with compatible dimensions, we have

$$
\begin{aligned}
& x(0)^{\prime} \hat{W}^{k}\left(I_{n}-\hat{W}^{k}\right) \mathbf{1}_{n}+\mathbf{1}_{n}^{\prime}\left(I_{n}-\hat{W}^{k}\right)^{\prime}\left(\hat{W}^{k}\right)^{\prime} x(0) \\
& \leq \alpha x(0)^{\prime} \hat{W}^{k}\left(\hat{W}^{k}\right)^{\prime} x(0)+\alpha^{-1} \mathbf{1}_{n}^{\prime}\left(I_{n}-\hat{W}^{k}\right)^{2} \mathbf{1}_{n} .
\end{aligned}
$$


It follows from (8), (9) and (10) that

$$
\begin{aligned}
\sum_{k=0}^{T} \| \hat{W}^{k} x(0) & +c\left(I_{n}-\hat{W}^{k}\right) \mathbf{1}_{n} \|_{2}^{2} \\
< & (1+c \alpha) x(0)^{\prime}\left(I_{n}-\hat{W}^{2}\right)^{-1} x(0) \\
& +\frac{1}{2} l\left(c^{2}+c \alpha^{-1}\right) T(T+1) .
\end{aligned}
$$

Note that the optimization variables are $\gamma_{i}$ 's. For any given $c$, $\alpha>0, T>0,0<l<\min _{i}\left\{w_{i i}\right\}$, the following optimization problem

$$
\begin{aligned}
& \min _{\Gamma}(1+c \alpha) x(0)^{\prime}\left(I_{n}-\hat{W}^{2}\right)^{-1} x(0)+\frac{1}{2} l\left(c^{2}+c \alpha^{-1}\right) T(T+1) \\
& \text { s.t. } \quad \gamma_{i} \in\{0,1\}, i=1, \ldots, n
\end{aligned}
$$

is equivalent to the problem (7). Both problems have identical feasible sets despite the different forms. As a result, the objective function in (6) has an upper bound, which is the right-hand side of (11).

By Theorem 1, we obtain the following relaxation of $\left(P_{0}\right)$ :

$$
\begin{aligned}
\left(P_{2}\right): \quad \min _{\Gamma} & p x(0)^{\prime}\left[\left(I_{n}+\hat{W}\right)^{-1}+\left(I_{n}-\hat{W}\right)^{-1}\right] x(0) \\
+ & (1-p) u(0)^{\prime}\left[\left(I_{n}+\hat{W}\right)^{-1}+\left(I_{n}-\hat{W}\right)^{-1}\right] u(0) \\
\text { s.t. } & \operatorname{tr}(\Gamma) \leq q, \\
& \gamma_{i} \geq\|1-\|(\hat{W})_{i}\left\|_{1}\right\|_{0}, i=1, \cdots, n,
\end{aligned}
$$

where $\|\cdot\|_{0}$ (called the $l_{0}$ norm) of a scalar is 0 if the scalar is 0 (it is 1 otherwise), $(\hat{W})_{i}$ denotes the $i$ th column of the matrix $\hat{W}$.

Remark 2 The main improvement of formulation $\left(P_{2}\right)$ over $\left(P_{0}\right)$ is that, instead of

$$
\sum_{k=0}^{T} p\|x(k)\|_{2}^{2}+(1-p)\|u(k)\|_{2}^{2},
$$

the objective function in $\left(P_{2}\right)$ is independent of the timehorizon and also shows the explicit relation with the $\gamma_{i}$ s. Moreover, each term of the objective function is a matrix fractional function with the form

$$
f(x, Y)=x^{T} Y^{-1} x,
$$

which is convex on $\operatorname{dom} f=\mathbb{R}^{n} \times \mathbb{S}_{++}^{n}$ (See, Example 3.4, [34]). However, the problem $P_{2}$ is still a combinatorial problem because the constraint on $\gamma_{i}$ contains the $l_{0}$ norm. In recent years, some heuristic methods have been proposed to replace $l_{0}$ norm by other norms like $l_{1}$ norm. Note that $\left(I_{n}+\hat{W}\right)^{-1}$ and $\left(I_{n}-\hat{W}\right)^{-1}$ are positive definite matrices. Thus, we can relax the problem $P_{2}$ to a convex optimization problem using the reweighted $l_{1}$ norm, which will be discussed in the next subsection.
Remark 3 Note that here we only discuss the nodes selection problem for fixed network topology. To the best of our knowledge, the studies for the nodes selection in a dynamical network topology are less. Recently, the work [25] considers a special random network, and discusses the random variables with known and unknown distribution, respectively. The challenge of analyzing the dynamic case mainly comes from the computation complexity, particularly in a large-scale network. If the controlled nodes set varies over time, then selecting the controlled nodes set for each topology in order to optimizing the objective function is equivalent to solving a series of static optimization problem. When compared with solving the optimization problem, it might be more feasible to use some heuristic methods to select the optimal nodes. In the future work, we will consider the nodes selection problem for some special dynamical network topology.

\subsection{Sub-optimal nodes selection algorithm}

For a large-scale network, the Boolean combinatorial problem $P_{2}$ becomes computationally infeasible since one has to check all possible results for nodes selection satisfying the constraints. In this subsection, we further relax the problem $P_{2}$ to a convex optimization problem, where the solutions are computationally feasible though suboptimal. We use the technique proposed in [35] to replace the $l_{0}$ norm with the $l_{1}$ norm. To avoid the main drawback that the larger numbers in $l_{1}$ are penalized much more than those in $l_{0}$, we replace the $l_{0}$ norm by a weighted $l_{1}$ norm which is similar to the work [36]. Using all the previous results, we propose an algorithm to solve the suboptimal problem $P_{2}^{\prime}$ relaxed by the problem $P_{2}$. The algorithm consists of four steps as follows.

Algorithm 1 The suboptimal nodes selection algorithm: fixed controlled nodes

1: Initialization: $z=0, f_{i}(0)=1$ for $i=1, \ldots, n$.

2: Solve the weighted $l_{1}$ minimization problem:

$$
\begin{aligned}
\left(P_{2}^{\prime}\right): \min _{\Gamma} & p x(0)^{\prime}\left[\left(I_{n}+\hat{W}\right)^{-1}+\left(I_{n}-\hat{W}\right)^{-1}\right] x(0) \\
& +(1-p) u(0)^{\prime}\left[\left(I_{n}+\hat{W}\right)^{-1}+\left(I_{n}-\hat{W}\right)^{-1}\right] u(0) \\
\text { s.t. } & \operatorname{tr}(\Gamma) \leq q \\
& \gamma_{i}(z) \geq f_{i}(z)\left(1-\left\|(\hat{W})_{i}\right\|_{1}\right), i=1, \cdots, n .
\end{aligned}
$$

Let the solution be $\gamma_{1}(z), \ldots, \gamma_{n}(z)$.

3: Update the weights:

$$
f_{i}(z+1)=\frac{1}{\gamma_{i}(z)+\delta}, \delta>0 .
$$

4: Terminate if either $z$ reaches a specified maximum number of iterations $z_{\max }$ or the solution has converged. Otherwise, increase $z$ and return to step 2 .

Since the matrix $\left(I_{n}+\hat{W}\right)$ and $\left(I_{n}-\hat{W}\right)$ are nonsingular symmetric, the procedure to compute its inverse use $O\left(\frac{1}{3} n^{3}\right)$ operations without exploiting structure. In problem $P_{2}^{\prime}$, the objective function can be computed using $O\left(\frac{2}{3} n^{3}+2 n^{2}+2 n\right)$ 
operations and the constraint of $\gamma_{i}(z), i=1, \ldots, n$ can be evaluated using $O\left(n^{2}\right)$ operations. Totally, computing Algorithm 1 requires $O\left(n^{3} z\right)$ operations. In large-scale networks, the computation complexity could be further reduced by exploiting structure of the underlying matrix $\hat{W}$, see [34].

Remark 4 In some real-world applications, we do not really require a very accurate solution because we need to obtain the threshold of $\gamma_{i}$ and make it binary. Usually the problem takes less number of reweightings for the solution to converge. Since a function is convex if and only if its epigraph is a convex set [34], we can represent the problem $P_{2}^{\prime}$ as a series of linear matrix inequalities (LMIs), which can be solved by many methods, for example, interior point and steepest descent method. Specifically, when the network scale is large, by using the alternating direction method of multipliers, the problem $P_{2}^{\prime}$ can be decomposed into a sequence of separate minimization problems which can be solved in parallel.

\subsection{Switched nodes selection problem}

As discussed above, we have relaxed the fixed nodes selection problem as a suboptimal convex optimization problem. In practice, node faults and communication disruptions occur frequently, and this motivates us to investigate the switched nodes selection problem, where the controlled nodes are switched at each time step.

Similar to the analysis for fixed nodes selection, we rewrite the cost function to be minimized as

$$
\begin{aligned}
& x(k)^{T} x(k)+u(k)^{T} u(k) \\
& \quad=x(k)^{T} x(k)+\left(c \mathbf{1}_{n}-x(k)\right)^{T}\left(c \mathbf{1}_{n}-x(k)\right) \\
& \quad=\left(c \mathbf{1}_{n}\right)^{T}\left(c \mathbf{1}_{n}\right)+2 x(k)^{T} x(k)-2\left(c \mathbf{1}_{n}\right)^{T} x(k) \\
& \quad=\left(c \mathbf{1}_{n}\right)^{T}\left(c \mathbf{1}_{n}\right)-2 u(k)^{T} x(k) .
\end{aligned}
$$

By induction, we have

$$
x(k)=W^{k} x(0)+l \sum_{i=0}^{k-1} W^{k-1-i} \Gamma(i) u(i) .
$$

Obviously, when $\Gamma(k)=I$, we have

$$
x^{\star}(k)=W^{k} x^{\star}(0)+l \sum_{i=0}^{k-1} W^{k-1-i} u^{\star}(i) .
$$

Given the initial states, $x^{\star}(k)$ can be obtained by iterative method.
Set $x(0)=x^{\star}(0)$. Using (13) and (14) yields

$$
\begin{aligned}
u(k)^{T} x(k) & \left(c \mathbf{1}_{n}-x(k)\right)^{T} x(k) \\
= & {\left[c \mathbf{1}_{n}-x^{\star}(k)-l \sum_{i=0}^{k-1} W^{k-1-i} \Gamma(i) u(i)+l \sum_{i=0}^{k-1} W^{k-1-i} u^{\star}(i)\right]^{T} } \\
& \cdot\left[x^{\star}(k)-l \sum_{i=0}^{k-1} W^{k-1-i} u^{\star}(i)+l \sum_{i=0}^{k-1} W^{k-1-i} \Gamma(i) u(i)\right] \\
= & u^{\star}(k)^{T} x^{\star}(k)+\Delta u(k-1)^{T}\left(u^{\star}(k)-x^{\star}(k)\right) \\
& -\Delta u(k-1)^{T} \Delta u(k-1)
\end{aligned}
$$

where

$$
\Delta u(k-1)=l \sum_{i=0}^{k-1} W^{k-1-i} \Gamma(i) u(i)-l \sum_{i=0}^{k-1} W^{k-1-i} u^{\star}(i) .
$$

Define

$$
\begin{aligned}
& \hat{W}(k-1)=\left[W^{k-1}, W^{k-2}, \cdots, I\right], \\
& \tilde{\Gamma}(k-1)=\operatorname{diag}(\Gamma(0), \Gamma(1), \cdots, \Gamma(k-1)), \\
& \tilde{u}(k-1)=\left[u(0)^{T}, u(1)^{T}, \cdots, u(k-1)^{T}\right]^{T}, \\
& \tilde{u}^{\star}(k-1)=\left[u^{\star}(0)^{T}, u^{\star}(1)^{T}, \cdots, u^{\star}(k-1)^{T}\right]^{T}
\end{aligned}
$$

and we have

$$
\Delta u(k-1)=l \hat{W}(k-1) \tilde{\Gamma}(k-1) \tilde{u}(k-1)-l \hat{W}(k-1) \tilde{u}^{\star}(k-1) .
$$

Using all the previous arguments, we rewrite the cost function as

$$
\begin{aligned}
& x(k)^{T} x(k)+u(k)^{T} u(k) \\
& =n c^{2}-2 u^{\star}(k)^{T} x^{\star}(k)-2 \Delta u(k-1)^{T}\left(c \mathbf{1}_{n}-2 x^{\star}(k)\right) \\
& \quad+2 \Delta u(k-1)^{T} \Delta u(k-1) .
\end{aligned}
$$

Thus, we can address the optimization problem $P_{1}$ as follows:

$$
\begin{aligned}
\left(P_{3}\right): \quad \min _{\substack{\tilde{\Gamma}(k-1) \\
\tilde{u}(k-1)}} \sum_{k=0}^{N T} n c^{2}-2 u^{\star}(k)^{T} x^{\star}(k) & \\
& -2 \Delta u(k-1)^{T}\left(c \mathbf{1}_{n}-2 x^{\star}(k)\right) \\
& +2 \Delta u(k-1)^{T} \Delta u(k-1) \\
\text { s.t. } & \operatorname{tr}(\Gamma(k)) \leq q, \\
& \gamma_{i}(k) \in\{0,1\}, i=1, \cdots, n, \\
& N T \\
& \sum_{k=1}^{N T} \gamma_{i}(k) \leq r_{i}, \\
& u(k+1)=(W-l \Gamma(k)) u(k) .
\end{aligned}
$$

Obviously, with the window size $N T$ increasing, the number of optimization variables grows rapidly at the rate of 
$\left(O\left(N^{2} T^{2}\right)\right)$. A heuristic method is to solve the optimization problem during each time interval $T$ which is similar to the greedy algorithm. This way, the solution is an approximation to the original problem, but the cost function with the constraints can be reformulated to a convex minimization problem by replacing the $l_{0}$ norm with the $l_{1}$ norm, and the computation complexity is largely reduced. In this subsection, we divide the whole time-horizon into several time-intervals $[1, T],[T+1,2 T], \cdots,[(N-1) T+1, N T]$. Let $\Gamma(k)$ be the node selection matrix. During each time interval, given the previous states $x(k-1)$ and $\Gamma(k-1)$, the suboptimal minimization problem over $\Gamma(k)$ of the cost function can be solved by using the following algorithm.

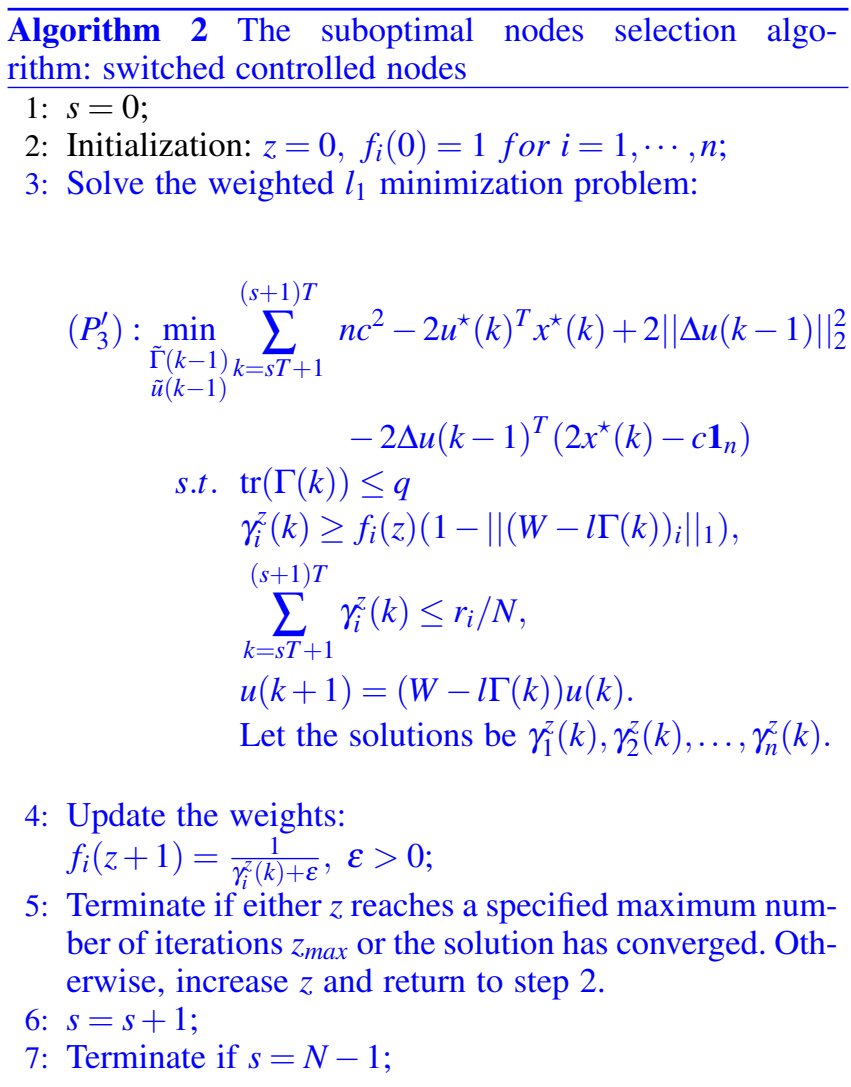

Remark 5 Recall that the objective function in $P_{3}^{\prime}$ includes the quadratic function $\|\Delta u(k)\|_{2}^{2}$. It can be computed using $O\left(2 n^{3}+4 n^{2}\right)$ operations. Thus the objective function over the window size $T$ requires $O\left(n^{3} T^{2}\right)$ operations. The constraint of $\gamma_{i}^{z}(k), i=1, \ldots, n$ can be evaluated using $O\left(n^{2}\right)$ operations. Hence, the complexity of Algorithm 2 is $O\left(n^{3} T^{2} N^{2}\right)$.

Using Algorithm 2, we can obtain a suboptimal solution to minimize the objective function. Subsequently, a question is how to measure the gap of $J(\Gamma(k))$ between the $P_{3}^{\prime}$ and $P_{3}$ problems. To answer this question, we first introduce two strategies. Let us denote $S_{1}$ the strategy for optimal nodes selection given by $P_{3}$ in $[1, N T]$. Let $J_{S_{1}}(k)=p\left\|x_{S_{1}}(k)\right\|_{2}^{2}+$ $(1-p)\left\|u_{S_{1}}(k)\right\|_{2}^{2}$ be the cost function at time $k$ given by the strategy $S_{1}$. We use the greedy algorithm to solve problem $\left(P_{3}^{\prime}\right)$ from time 1 to time $T$ and then solve it again for time $T+1$ to $2 T$ and so on. Again, denote $S_{2}$ the nodes selection strategy in $[1, N T]$ given by the greedy method with $J_{S_{2}}(k)$ being the corresponding cost matrix.

Theorem 2 The gap of strategy $S_{2}$ with respect to $S_{1}$ satisfies the following inequality:

$$
\sum_{k=1}^{N T} \operatorname{tr}\left(J_{S_{2}}(k)\right)-\sum_{k=1}^{N T} \operatorname{tr}\left(J_{S_{1}}(k)\right) \leq(N-1) \frac{\rho \beta}{\theta}
$$

where

$$
\begin{aligned}
\rho & =\inf \left\{h>0 \mid J_{S_{2}}(i T) \preceq(1+h) J_{S_{1}}(i T), i=1, \cdots, N-1\right\}, \\
\beta & =\sup \left\{\operatorname{tr}\left(J_{S_{1}}(k)\right) \mid k=T+1, \cdots, N T\right\}, \\
\theta & =\sup \left\{h>0 \mid \rho\left(p l^{2} \Gamma(k) c \mathbf{1}(c \mathbf{1})^{\prime} \Gamma(k)-\delta I\right)-2 \delta I\right. \\
& \left.\succeq h\left(\rho J_{S_{1}}(k)+2 \delta I\right)\right\}, k=T+1, \ldots, N T, \\
\delta & =\sup \left\{h>0 \mid p l \alpha^{-1}\left\|(W-l \Gamma(k)) x(k) x(k)^{\prime}(W-l \Gamma(k))^{\prime}\right\|_{2}\right. \\
& \leq h, k=T+1, \ldots, N T .\}
\end{aligned}
$$

Proof: Similar to [36], let us define a third nodes selection strategy $S_{3}$ as:

$$
\begin{aligned}
& S_{3}[1, T]=S_{2}[1, T] \\
& S_{3}[T+1,2 T]=S_{1}[T+1,2 T] .
\end{aligned}
$$

By the definition of the three strategies, we know that, from time 1 to $T, S_{2}$ is the optimal strategy and therefore,

$$
\sum_{k=1}^{T} \operatorname{tr}\left(J_{S_{3}}(k)\right)=\sum_{k=1}^{T} \operatorname{tr}\left(J_{S_{2}}(k)\right) \leq \sum_{k=1}^{T} \operatorname{tr}\left(J_{S_{1}}(k)\right) .
$$

From time $T+1$ to time $2 T$, the strategy $S_{2}$ is the optimal for initial condition $J_{S_{2}}(T)$, and the strategy $S_{3}$ has the same initial condition as $S_{2}$, i.e., $J_{S_{2}}(T)=J_{S_{3}}(T)$. Therefore,

$$
\sum_{k=T+1}^{2 T} \operatorname{tr}\left(J_{S_{3}}(k)\right) \geq \sum_{k=T+1}^{2 T} \operatorname{tr}\left(J_{S_{2}}(k)\right) .
$$

Furthermore, one obtains

$$
\begin{aligned}
& \sum_{k=1}^{2 T} \operatorname{tr}\left(J_{S_{2}}(k)\right)-\sum_{k=1}^{2 T} \operatorname{tr}\left(J_{S_{1}}(k)\right) \\
& \leq \sum_{k=T+1}^{2 T} \operatorname{tr}\left(J_{S_{3}}(k)\right)-\sum_{k=T+1}^{2 T} \operatorname{tr}\left(J_{S_{1}}(k)\right) .
\end{aligned}
$$


Starting at time $T$, the cost matrix at time $T+1$ is

$$
\begin{aligned}
p x & (T+1) x(T+1)^{\prime}+(1-p) u(T+1) u(T+1)^{\prime} \\
= & (W-l \Gamma(T))\left[p x(T) x(T)^{\prime}+(1-p) u(T) u(T)^{\prime}\right](W-l \Gamma(T))^{\prime} \\
& +p l(W-l \Gamma(T)) x(T)(c \mathbf{1})^{\prime}(\Gamma(T))^{\prime} \\
& +p l \Gamma(T) c \mathbf{1} x(T)^{\prime}(W-l \Gamma(T))^{\prime}+p l^{2} \Gamma(T) c \mathbf{1}(c \mathbf{1})^{\prime} \Gamma(T) \\
\preceq & (W-l \Gamma(T))\left[p x(T) x(T)^{\prime}+(1-p) u(T) u(T)^{\prime}\right](W-l \Gamma(T))^{\prime} \\
& +p l \alpha^{-1}(W-l \Gamma(T)) x(T) x(T)^{\prime}(W-l \Gamma(T))^{\prime} \\
& +\left(p l^{2}+p l \alpha\right) \Gamma(T) c \mathbf{1}(c \mathbf{1})^{\prime} \Gamma(T) \\
\preceq & (W-l \Gamma(T))\left[p x(T) x(T)^{\prime}+(1-p) u(T) u(T)^{\prime}\right](W-l \Gamma(T))^{\prime} \\
& +\delta I+p l^{2} \Gamma(T) c \mathbf{1}(c \mathbf{1})^{\prime} \Gamma(T)
\end{aligned}
$$

where the second inequality follows from the fact that $\left(\alpha^{\frac{1}{2}} x-\alpha^{-\frac{1}{2}} y\right)\left(\alpha^{\frac{1}{2}} x-\alpha^{-\frac{1}{2}} y\right)^{\prime} \geq 0$. Note that $\alpha$ is a positive real and $x(k)$ will converge to a constant vector. Thus, we can always find a sufficient large $\alpha$ such that $p l \alpha^{-1}\left\|(W-l \Gamma(T)) x(T) x(T)^{\prime}(W-l \Gamma(T))^{\prime}\right\|_{2} \leq \delta$.

Similarly, we have

$$
\begin{gathered}
p x(T+1) x(T+1)^{\prime}+(1-p) u(T+1) u(T+1)^{\prime} \succeq \\
\begin{array}{c}
(W-l \Gamma(T))\left[p x(T) x(T)^{\prime}+(1-p) u(T) u(T)^{\prime}\right](W-l \Gamma(T))^{\prime} \\
-\delta I+p l^{2} \Gamma(T) c \mathbf{1}(c \mathbf{1})^{\prime} \Gamma(T) .
\end{array}
\end{gathered}
$$

Using (15) and (16), we obtain

$$
\begin{aligned}
& J_{S_{3}}(T+1) \\
& \preceq(W-l \Gamma(T)) J_{S_{3}}(T)(W-l \Gamma(T))^{\prime}+\delta I+p l^{2} c \mathbf{1}(c \mathbf{1})^{\prime} \Gamma(T) \\
& \preceq(1+\rho)(W-l \Gamma(T)) J_{S_{1}}(T)(W-l \Gamma(T))^{\prime} \\
& \quad+\delta I+p l^{2} c \mathbf{1}(c \mathbf{1})^{\prime} \Gamma(T) \\
& \preceq\left(1+\frac{\rho}{1+\theta}\right) J_{S_{1}}(T+1) .
\end{aligned}
$$

By induction, we have

$$
J_{S_{3}}(T+k) \preceq\left(1+\frac{\rho}{(1+\theta)^{k}}\right) J_{S_{1}}(T+k), k=1, \ldots, T,
$$

and furthermore

$$
\operatorname{tr}\left(J_{S_{3}}(T+k)\right) \leq\left(1+\frac{\rho}{(1+\theta)^{k}}\right) \operatorname{tr}\left(J_{S_{1}}(T+k)\right), k=1, \ldots, T .
$$

As a result, we have

$$
\begin{aligned}
\sum_{k=T+1}^{2 T} \operatorname{tr}\left(J_{S_{3}}(k)\right)- & \sum_{k=T+1}^{2 T} \operatorname{tr}\left(J_{S_{1}}(k)\right) \\
& \leq \sum_{k=1}^{T}\left(1+\frac{\rho}{(1+\theta)^{k}}\right) \operatorname{tr}\left(J_{S_{1}}(T+k)\right) \\
& \leq \sum_{k=1}^{T} \frac{\rho \beta}{(1+\theta)^{k}} \leq \frac{\rho \beta}{\theta}
\end{aligned}
$$

The proof is now complete.

Remark 6 If $N=1$, then the strategy $S_{2}$ is equal to the optimal strategy $S_{1}$ and the inequality is relaxed to

$$
\sum_{k=1}^{N T} \operatorname{tr}\left(J_{S_{2}}(k)\right)=\sum_{k=1}^{N T} \operatorname{tr}\left(J_{S_{1}}(k)\right)
$$

\section{Numerical examples}

In this section, we evaluate the performance of the proposed strategy for the nodes selection problem via simulation study. We choose an undirected network with $n=10$ nodes as shown in Fig. 1, which has its second largest eigenvalue $\lambda_{n-1}(W)=0.9429$ and maximal degree $\Delta=4$. We also choose $c=[3,2]^{\prime}, l=0.1, r_{i}=50, i=1,2, \ldots, n$.

First, we study the selection of fixed controlled node in the case when $q=1$. Fig. 2 shows the value of objective function $J$ varying with the degree of controlled node over window size $T=300$. By using Algorithm 1 , when $p=1$, we find that the selected node having the lowest degree (nodes 5,7 ) leads to the minimum value $J^{\star}$; when $p=0$, we find that the selected node having the highest degree (node 6) leads to the minimum value $J^{\star}$. The heuristic reason is that the node with higher degree can spread the information to the whole network with faster convergence speed as quantified by $\|u(k)\|_{2}^{2}$, and the node with lower degree is affected by fewer adjacent nodes which, in turn, results in the fewer energy of control action as quantified by $\|x(k)\|_{2}^{2}$.

Moreover, it is worth mentioning that nodes 2,3,10 also have 4 neighboring nodes, while the optimal controlled node is node 6 when $p=0$. It can be seen that node 6 has the maximal betweenness centrality. Here, Betweenness centrality is an indicator of a node's centrality in a network. It is equal to the number of shortest paths from all nodes to all others that pass through that node. A node with high betweenness centrality has a large influence on the transfer of items through the network, under the assumption that item transfer follows the shortest paths (see [37]). To further study the influence of node centrality on the performance index, we compare the value of objective function under different selection strategies. As Fig. 3 shows, the strategy of $b c_{\max }$ approaches to the optimal one when $p<0.3$, and the strategy of $b c_{\text {min }}$ approaches to the optimal one when $p>0.4$. The heuristic reason is that the smaller $p$, the term $u(k)^{\prime} u(k)$ dominates the objective function much more. Note that the betweenness centrality reflects the rate of transferring items through the network. Thus, when $p$ is relatively small, the node with maximal betweenness centrality leads to the minimal $J$; while when $p$ is relatively large, the node with minimal betweenness centrality leads to the minimal $J$. These results also further show that a tradeoff exists between the energy of control actions and the convergence speed, and the betweenness centrality could be an index to select the controlled node efficiently. Moreover, the proposed suboptimal strategy is closer to the optimal one when compared 
with the other strategies, which verifies the effectiveness of the proposed suboptimal strategy.

Next, let us investigate the relationship between the minimum value $J^{\star}$ and the weight value $p$ for various values of $q$. In Fig. 4, each point corresponds to a minimum value $J^{\star}$ for a fixed $p$ under the constraint $\operatorname{tr}(\Gamma)=q$. Obviously, for an arbitrary $q$, the minimum value $J^{\star}$ increases as the increasing $p$. Notice that the convergence speed quantified by $\|u(k)\|_{2}^{2}$ dominates the objective function when $p$ is relatively small, while $\|x(k)\|_{2}^{2}$ dominates the objective function when $p$ is relatively large. As we can observe, when $p<0.2$, the larger $q$ leads to the smaller $J^{\star}$ for a fixed $p$, which implies that much more nodes selected as the controlled nodes result in faster convergence speed. Conversely, when $p>0.4$, the smaller $q$ leads to the smaller $J^{\star}$ for a fixed $p$, which implies that the fewer nodes selected as the controlled nodes results in the fewer energy of control actions. Based on these results, we suggest that there exists two critical values for $p$, the lower bound $\check{p}$ and the upper bound $\hat{p}$, which depends on the node dynamics and network topology. To solve the problem $P_{0}$, when $p \leq \check{p}$, the minimum value $J^{\star}$ can be achieved by selecting $q$ optimal nodes, that is to say, the constraint $\operatorname{tr}(\Gamma) \leq q$ can be reduced to $\operatorname{tr}(\Gamma)=q$. When $p \geq \hat{p}$, the minimum value $J^{\star}$ can be achieved by selecting a single optimal node, that is to say, the constraint $\operatorname{tr}(\Gamma) \leq q$ can be reduced to $\operatorname{tr}(\Gamma)=1$. However, when $\check{p}<p<\hat{p}$, the optimal solutions for the number of controlled nodes are not explicit, which can only be obtained by solving the problem $P_{0}$.

It can be seen from Fig. 5 that the gap of the minimum value $J^{\star}$ between two strategies varies with $q$ over the window size $T=4000$. In the case when the controlled nodes are fixed, the gap of $J^{\star}$ between the proposed suboptimal strategy and the optimal one decreases as the increasing $q$ (dotted green line). Specifically, when $q$ is above a critical value, the gap is close to zero. Similarly, in the case when the controlled nodes switch, the gap between the strategy of optimizing over the whole time horizon and one of optimizing over the small intervals is investigated, which converges to zero as well. Aiming at the above results, the following heuristic analysis are provided. First, let us define the settling time when all the nodes reach desired consensus, denoted by $k_{s}$. For an arbitrary $q$, when $k \geq k_{s}$, the value of objective function equals to $n p c^{\prime} c$. Thus, the gap accumulates during the time horizon $k<k_{s}$. Intuitively, the smaller $k_{s}$, the smaller gap. Recall that the larger $q$ leads to the smaller $k_{s}$, which has been shown in Fig. 2. On the other side, as $q$ increases, the probability of selecting those optimal controlled nodes increases as well, which also leads to the gap converging to zero.

\section{Conclusions}

In this paper, we have considered the selection problem of controlled nodes with the goal of minimizing the objective function that caters both the energy of control actions and the convergence speed. We have addressed the nodes selection

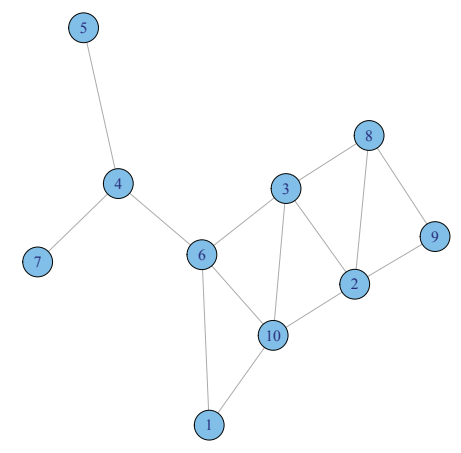

Fig. 1. The network topology

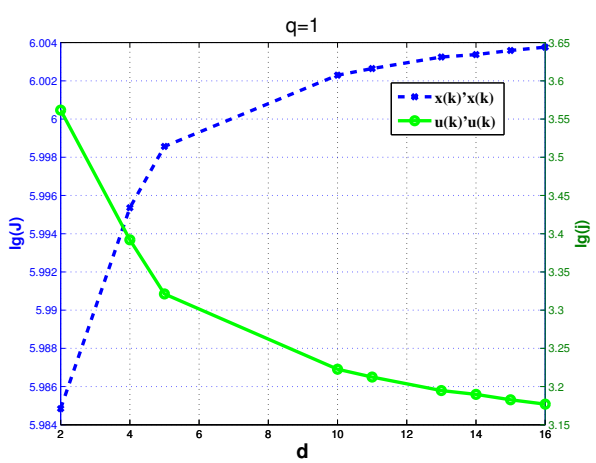

Fig. 2. The value of objective function $\lg (J)$ varies as the degree of controlled node. Here, the value of $J$ is calculated by adding all the values of $x(k)^{\prime} x(k)$ or $u(k)^{\prime} u(k)$ from $k=0$ to $k=300$. The blue dashed line is corresponding to the energy of control action quantified by $\|x(k)\|_{2}^{2}$, i.e., $p=1$; the red line is corresponding to the convergence time quantified by $\|u(k)\|_{2}^{2}$, i.e., $p=0$.

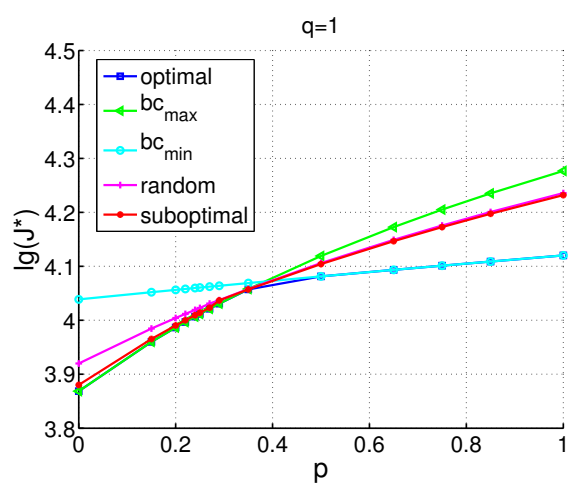

Fig. 3. The value of objective function $\lg (J)$ varies as the weight value $p$ under different selection strategies. $b c_{\max }$ and $b c_{\min }$ : the strategy of selecting the node with maximal and minimal betweenness centrality; random: the strategy of selecting a node randomly; suboptimal: the strategy with Algorithm 1.

as a combinatorial optimization problem, and further reformulated it using a convex relaxation based on a reweighed $l_{1}$ approximation. In the case when the controlled nodes are fixed, we have obtained an upper bound of the objective function in order to make the optimization independent of time horizon, which is also shown to be convex. In the 


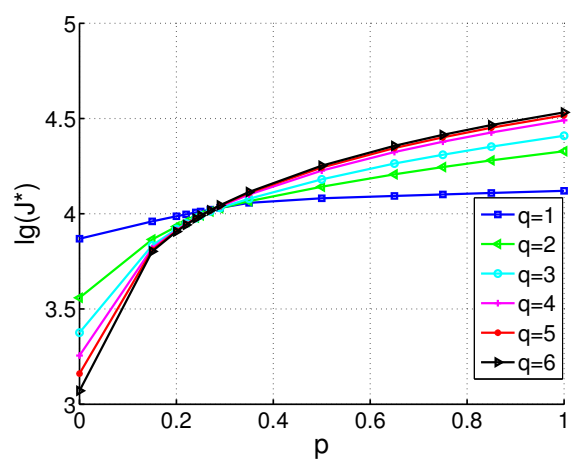

Fig. 4. The minimum of the objective function $\lg \left(J^{\star}\right)$ vs. $p$ for various values of $q$.(Here, we set the constraint in $P_{0}$ as $\operatorname{tr}(\Gamma)=\mathrm{q}$.)

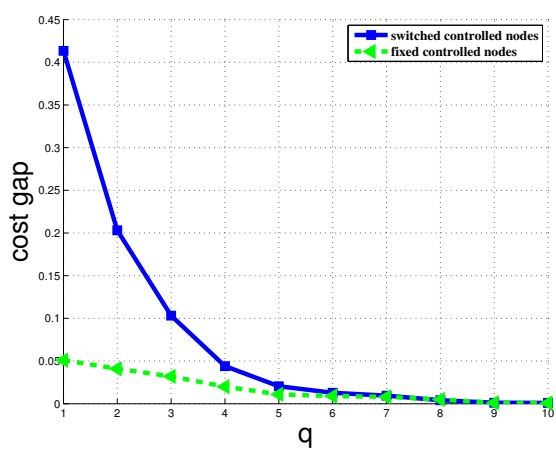

Fig. 5. The cost gap vs. $q(p=0.3)$. The blue line: the gap of $J^{\star}$ between the strategy of optimizing over the whole time horizon and the strategy of optimizing over the small intervals (switched controlled nodes); The green dotted line: the gap of $J^{\star}$ between the suboptimal strategy and the optimal one (fixed controlled nodes).

case when the controlled nodes switch, we have introduced a greedy algorithm to solve the optimization problem over many small time intervals. We have also established the relationship of the network parameters and the gap of minimum objective function between the strategy of optimizing over the whole time-horizon and the strategy of optimizing over small time intervals. In the end, the simulation results have verified the effectiveness of the proposed strategy. It has been shown that, when the weight value $p$ is less than a lower bound or greater than an upper bound, the number of the optimal controlled nodes is determined. The gap of the minimal objective function has also been shown to be decreasing as the number of controlled nodes increases.

\section{References}

[1] Fax, A., \& Murray, R. M. (2004). Information flow and cooperative control of vehicle formations, IEEE Trans. Autom. Contr., 49(9), 1465-1476.

[2] Jadbabaie, A., Lin, J., \& Morse, A. (2003). Coordination of groups of mobile autonomous agents using nearest neighbor rules, IEEE Trans. Autom. Contr., 48(6),988-1001.

[3] Olfati-Saber, R., \& Murray, R. M. (2004). Consensus problems in networks of agents with switching topology and time-delays, IEEE Trans. Autom. Contr., 49(9),1520-1533.
[4] Moreau, L. (2005). Stability of multiagent systems with timedependent communication links, IEEE Trans. Autom. Contr., 50(2), 169-1825.

[5] Ren, W., \& Beard, R. W. (2005). Consensus seeking in multi-agent systems using dynamically changing interaction topologies, IEEE Trans. Autom. Contr., 50(5),655-661.

[6] Cao, M., Morse, A. S., \& Anderson, B. D. O. (2008). Reaching a consensus in a Dynamically changing environment: convergence rates, measurement delays, and asynchronous events, SIAM J. Control Optim., 47(2),601-623.

[7] Xiao, F., \& Wang, L. (2008). Asynchronous consensus in continuoustime multi-agent systems with switching topology and time-varying delays, IEEE Trans. Autom. Contr., 53(8), 1804-1816.

[8] Xiao, L., \& Boyd, S. (2004). Fast linear iterations for distributed averaging, Syst. Control Lett., 53(1), 65-78.

[9] Xiao, L., Boyd, S., \& Kim, S. J. (2007). Distributed average consensus with least-mean-square deviation, J. Parallel Distr. Com., 67(1), 33-46.

[10] Tanner, H. G. (2004). On the controllability of nearest neighbor interconnections, in: Proceedings of the 43rd IEEE Conference on Decision and Control, 2467-2472.

[11] Liu, B., Chu, T., Wang, L., \& Xie, G. (2008). Controllability of a leader-follower dynamic network with switching topology, in: IEEE Trans. Autom. Control, 53(4), 1009-1013.

[12] Ji, Z., Wang, Z., Lin, H., \& Wang, Z. (2009). Interconnection topologies for multi-agent coordination under leader-follower framework Automatica, 45(12), 2857-863.

[13] Porfiri, M., \& Bernardo, M. D. (2008). Criteria for global pinningcontrollability of complex networks, Automatica, 44(12), 3100-3106.

[14] Schuler, S., Li, P., Lams, J., \& Allgöwar, F. (2011). Design of structured dynamic output-feedback controllers for interconnected systems, International Journal of Control, 84(12), 2081-2091.

[15] Yoon, M.G., \& Tsumura, K. (2011). Transfer function representation of cyclic consensus systems, Automatica, 47(9),1974-1982.

[16] Jafari, S., Ajorlou, A., \& Aghdam, A.G. (2011). Leader localization in multi-agent systems subject to failure: A graph-theoretic approach, Automatica, 47(8), 1744-1750.

[17] Parlangeli, G., \& Notarstefano, G. (2012). On the reachability and observability of path and cycle graphs, IEEE Trans. Autom. Contr., 57(3), 743-748.

[18] Rahmani, A., Ji, M., Mesbahi, M., \& Egerstedt, M. (2009). Controllability of multi-agent systems from a graph-theoretical perspective, SIAM J. Control Optim, 48(1),162-186.

[19] Egerstedt, M., Martini, S., Cao, M., Camlibel, K., \& Bicchi, A. (2012). Interacting with Networks: How does structure relate to controllability in single-leader, consensus networks? IEEE control systems magazine, 32(4), 66-73.

[20] Commault, C, Dion, J. M. (2013). Input addition and leader selection for the controllability of graph-based systems, Automatica, 49(11), 3322-3328.

[21] Porfiri, M., \& Florilli, F. (2009). Node-to-node pinning control of complex networks, Chaos, 19(1), 013112-013123.

[22] Patterson, S., \& Bamieh, B. (2010). Leader selection for optimal network coherence, in Proc. 49th IEEE Conf. Decision Control, 2692-2697.

[23] Kawashima, H., \& Egerstedt, M. (2012). Leader selection via the manipulability of leader-follower networks, in Proc. Amer. Control Conf., 6053-6058.

[24] Clark, A., \& Poovendran, R. (2011). A submodular optimization framework for leader selection in linear multi-agent systems, in Proc. 50th IEEE Conf. Decision Control Eur. Control Conf., 3614-3621. 
[25] Clark, A., Bushnell, L., \& Poovendran, R. (2014). A supermodular optimization framework for leader selection under link noise in linear multi-agent systems, IEEE Trans. Autom. Control, 59(2), 283-296.

[26] Clark, A., Alomair, B., Bushnell, L.,\& Poovendran, R. (2014). Minimizing convergence error in multi-agent systems via leader selection: a super modular optimization approach, IEEE Transactions on Automatic Control, 59(6), 1480-1494.

[27] Lin, F., Fardad, M., \& Jovanovic, M. R. (2014). Algorithms for leader selection in stochastically forced consensus networks, IEEE Transactions on Automatic Control, 59(7), 1789-1802.

[28] Li, X., Wang, X.F., \& Chen, G. R. (2004). Pinning a complex dynamical network to its equilibrium, IEEE Trans. Circuits Systs-I: Regular Papers, 51(10), 2074-2087.

[29] Zhou, J., Lu, J.A., \& Lü, J. H. (2006). Adaptive synchronization of an uncertain complex dynamical network, IEEE Trans. Autom. Contr., 51(4), 652-656.

[30] Zhou, J., Lu, J.A., \& Lü, J. H. (2008). Pinning adaptive synchronization of a general complex dynamical network, Automatica, 44(4), 996-1003.

[31] Chen, F., Chen, Z. Q., Xiang, L.Y., Liu, Z. X., \& Yuan, Z. Z. (2009). Reaching a consensus via pinning control, Automatica, 45(5), 12151220.

[32] Joshi, S., \& Boyd, S. (2009). Sensor selection via convex optimization, IEEE Trans. Sig. Proc., 57(2), 451-462.

[33] Horn, R. A., \& Johnson, C. A. (1985). Matrix analysis, UK: Cambridge University Press.

[34] Boyd, S., \& Vandenberghe, L. (2009). Convex Optimization, UK: Cambridge University Press.

[35] Candes, E. J., Wakin, M. B., \& Boyd, S. (2008). Enhancing sparsity by reweighted $l_{1}$ minimization. Journal of Fourier Analysis and Applications, 14(5), 877-905.

[36] Mo, Y. L., Ambrosino, R., \& Sinopoli, B. (2011). Sensor selection strategies for state estimation in energy constrained wireless sensor networks. Automatica, 47(7), 1330-1338.

[37] Newman, M.E.J. (2010). Networks: An Introduction. Oxford, UK: Oxford University Press. 